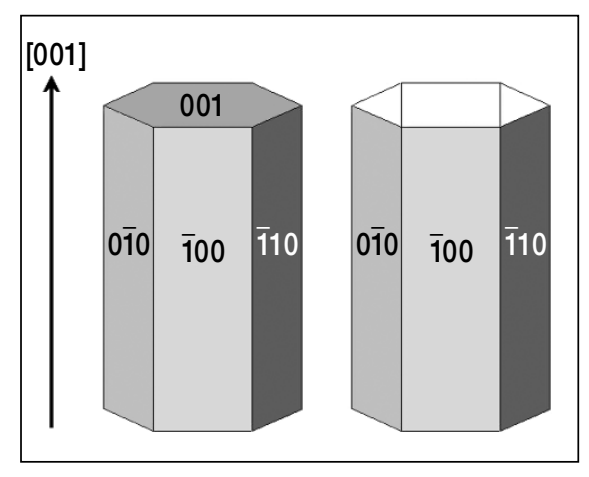

Figure. Crystal faces of hexagonal $\mathrm{ZnO}$ microrods and microtubes. Reprinted with permission from Chem. Mater., December 17, 2001, 13, 4395-4398. Copyright 2001 American Chemical Society.

Physical Chemistry at the University of Uppsala, Sweden, have prepared highly ordered arrays of hollow crystalline $\mathrm{ZnO}$ microtubes 1-2 $\mu \mathrm{m}$ in diameter and $10 \mu \mathrm{m}$ in length. The preparation, carried out by Lionel Vayssieres and co-workers, consisted of the growth of oriented crystalline $\mathrm{ZnO}$ microrods by controlled precipitation of $\mathrm{ZnO}$, and the selective etching of these rods to produce microtubes. The one-step synthesis was carried out in aqueous solution using common reagents and without the use of a template. This synthetic method allows for a simple, safe, and inexpensive production of purposebuilt materials on a variety of substrates. According to the researchers, similar microrod arrays have demonstrated high-UV photoresponse and excellent electron-transfer properties with potential applications as catalysts, sensors, and other photovoltaic devices.

As reported in the December 17, 2001, issue of Chemistry of Materials, the researchers prepared the microtube arrays by thermal decomposition of a $\mathrm{Zn}^{2+}$ methenamino complex precursor in aqueous solution to produce $\mathrm{ZnO}$. The arrays were grown on polycrystalline $\mathrm{F}-\mathrm{SnO}_{2}$ glass, silicon wafers, and indium-tinoxide-coated polyester substrates. The substrates were covered with the precursor solution, and the reaction was kept at $90^{\circ} \mathrm{C}$ for two days. The precursor solution was prepared by dissolving $\mathrm{Zn}\left(\mathrm{NO}_{3}\right)_{2}{ }^{*}$ $4 \mathrm{H}_{2} \mathrm{O}$ and methenamine (both $0.1 \mathrm{M}$ ) in MilliQ + and water. Following the synthesis, the product materials were washed with water to remove any unreacted complex and salts. Microtube arrays covering several tens of square centimeters may be produced by this method.
The formation of the microtubes in the arrays exploits the chemical and structural metastability of the (001) face of the $\mathrm{ZnO}$ wurtzite structure. A $\mathrm{ZnO}$ microrod consists of a hexagonal single crystal elongated along the [001] direction (Figure, left). This elongation is the result of preferential growth of the (001) face during conditions of high precursor concentration on the first day of the synthesis. When the precursor concentration drops, the resultant Ostwald ripening, combined with the preferential dissolution of the (001) face, results in a net transfer of material from the (001) face to the more stable crystal faces, producing a hollow structure (Figure, right side).

Scanning electron microscopy of the product materials showed a uniform array of hexagonal microtubes 1-2 $\mu \mathrm{m}$ in diameter and $10 \mu \mathrm{m}$ in length, oriented perpendicular to the substrate. $X$-ray and electron-diffraction studies confirm that the microtubes are crystalline and exhibit the wurtzite structure and lattice spacing of bulk $\mathrm{ZnO}$. The crystallinity of the arrays allows the use of flexible and temperaturesensitive substrates by eliminating the need for high-temperature annealing normally required for materials grown at low temperatures.

GREG KHITROV

\section{Array-to-Array Transfer of Microspheric Sensors Stable over Time}

Researchers from Tufts University's Max Tishler Laboratory for Organic Chemistry and the Johns Hopkins University's Department of Mathematical Sciences have devised a method to manufacture artificial noses by using microsensors that are typically $3 \mu \mathrm{m}$ in diameter. Due to the sensors' small size, literally billions of them can be made from the same feedstock. The microsensors are made of silica microspheres or polymer beads of variations of polystyrene. The microsensors are unique from other artificial-nose-array constituents in that they are relatively simple to make reproducibly, which allows for basically the same classifier training, an involved process typically done separately for each artificial nose array, to be used for all artificial-nose arrays made from the same baseline materials. The researchers said, "A conservative estimate puts the number of nose arrays made from $1 \mathrm{~g}$ of $3-\mu \mathrm{m}$ feedstock at 10,000 . Thus, we have essentially eliminated the need to retrain the classifier, thereby saving time and increasing accuracy."

As reported in the November 1, 2001, issue of Analytical Chemistry, the researchers led by David Walt at Tufts
University used 3- $\mu \mathrm{m}$ IB-Sil (C1), 3- $\mu \mathrm{m}$ Phenosphere $(\mathrm{OH}), 3-\mu \mathrm{m}$ Luna $(\mathrm{OH})$, and 5 - $\mu \mathrm{m}$ Selectosil (SCX) silica microspheres; in addition to polymer beads of 3.12- $\mu \mathrm{m}$ $\mathrm{P}(\mathrm{S} / 55 \%$ divinylbenzene) and the copolymer PS802. The silica or polymer beads were first dipped in Nile red (a fluorescent indicator) and dyed in a batch process using fluorescent materials. Next, the sensors were placed in a vacuum-filtration system before being rinsed with toluene or chloroform to remove excess dye. In the final step, the researchers dried the sensors in a $100^{\circ} \mathrm{C}$ oven for $\sim 1 \mathrm{~h}$. The sensors were stored in the dark prior to use.

Once the dye process was complete, the researchers made a slurry of the five different bead/sphere types, which they then sandwiched between two glass cover slides. In addition to making these randomized arrays, the researchers also made five different arrays from each type of microsensor, in order to determine the characteristics of each. Using a fluorescence imaging system based upon a charge-coupled device camera, the nose arrays were tested for their ability to detect explosive nitroaromatic-compound (NAC) vapors in the presence of other volatile organic compounds (VOC). NAC vapor sources included 1,3-dinitrobenzene and 4-nitrotoluene. VOC vapor sources included acetone, benzene, chloroform, ethanol, ethyl acetate, heptane, methanol, toluene, and air carrier gas (control). Fluorescence was excited at $530 \mathrm{~nm}$ and monitored at $630 \mathrm{~nm}$. Maps of sensor type were obtained by characterizing sensor interaction with air. Changes in fluorescence were then monitored for each sensor type upon exposure to other test samples. The researchers made many of the vapor mixtures overwhelmingly VOC, yet the artificial-nose arrays were almost always able to detect the NAC vapors. Indeed, the first artificial-nose array tested had a $98.2 \%$ correct classification rate, and the second nose array tested six months later had a $93.8 \%$ correct classification rate. Thus, these tests showed that the artificial-nose arrays produced using this scheme were not only accurate, but also stable over time.

PAMELA JOHNSON

\section{A Particle Constrained in an Optical Trap Allows 3-D Imaging of Transparent Materials}

Three-dimensional imaging of transparent materials can be achieved with a scanning probe microscope based on a dielectric particle trapped by optical tweezers. Its resolution is affected by thermal variations of the particle within the optical trap since the forces acting on this dielectrical particle are weaker than those 